\title{
TITLE:
}

\section{Critical behaviors of photoinduced giant permittivity in potassium tantalate}

$\operatorname{AUTHOR}(S):$

Katayama, I; Ichikawa, Y; Tanaka, K

\section{CITATION:}

Katayama, I ...[et al]. Critical behaviors of photoinduced giant

permittivity in potassium tantalate. PHYSICAL REVIEW B 2003, 67(10):

100102.

ISSUE DATE:

2003-03-01

URL:

http://hdl.handle.net/2433/50046

RIGHT:

Copyright 2003 American Physical Society 


\title{
Critical behaviors of photoinduced giant permittivity in potassium tantalate
}

\author{
Ikufumi Katayama, ${ }^{1, *}$ Yuki Ichikawa, ${ }^{1}$ and Koichiro Tanaka ${ }^{1,2}$ \\ ${ }^{1}$ Department of Physics, Graduate School of Science, Kyoto University, Sakyo-ku, Kyoto 606-8502, Japan ${ }^{\dagger}$ \\ ${ }^{2}$ Precursory Research for Embryonic Science and Technology (PRESTO), Japan Science and Technology Corporation (JST), Japan
}

(Received 12 August 2002; published 24 March 2003)

\begin{abstract}
We have demonstrated that a static permittivity of potassium tantalate is dramatically enhanced by band-toband excitation at low temperatures. A critical behavior has been observed in the relaxation time and the oscillator strength of its Debye-type dispersion. Two models, considering photoinduced mesoscopic clusters formed around excited carriers, can successfully reproduce temperature and carrier-density dependence of the photoinduced effect observed in the experiment.
\end{abstract}

DOI: 10.1103/PhysRevB.67.100102

PACS number(s): 71.38.-k, 77.90.+k, 78.20.-e

Photoinduced phase transition has been studied extensively in this decade as a typical phenomenon illustrating a cooperative interaction between excited states. ${ }^{1}$ In the field of magnetism, it has been confirmed recently that a structure of a photoinduced phase in the Fe spin-crossover complex is quite different from that of the thermally induced phase. ${ }^{2}$ This result opens a general possibility that light irradiation may induce a new macroscopic phase.

In the present study, we search for a novel macroscopic phase in inorganic dielectric materials under light irradiation. A common feature in previous studies is that light excitation induces a macroscopic quasistable ordering against thermal fluctuations. However, in many cases, we can also obtain these photoinduced phases thermally by heating the sample. Therefore, the focus is now on materials that quantum fluctuations suppress the ordering, as is the case of liquid helium. Quantum paraelectrics (QPE) is one such material, in which quantum fluctuations of the lattice prohibit a ferroelectric phase transition. Since electronic states of the dielectrics directly couple to the lattice properties, ${ }^{3}$ we can expect a strong photoinduced effect against the quantum fluctuations in the dielectric materials. Actually, several authors have demonstrated photoexcited carriers stabilize the polar clusters in dielectrics $^{4-7}$. In our experiment, we attempted to excite a new phase in QPE through photoexcitation and succeeded in observing drastic enhancement of its photoinduced permittivity as well as critical behaviors.

We employed typical quantum paraelectric potassium tantalate $\left(\mathrm{KTaO}_{3}\right)$ for experiments, which maintains a cubic perovskite structure at temperatures as low as $2 \mathrm{~K}$ without any structural phase transitions. $\mathrm{KTaO}_{3}$ crystals were grown by the spontaneous nucleation technique. ${ }^{8}$ A typical dimension of the sample was about $11 \times 3 \times 0.7 \mathrm{~mm}^{3}$. A pair of gold electrodes was evaporated onto a polished surface with $1 \mathrm{~mm}$ in separation and $4 \times 2.5 \mathrm{~mm}^{2}$ in dimension. We measured a permittivity using both HP 4284A and HIOKI 3531 LCR meters in a frequency range of $50 \mathrm{~Hz}-1 \mathrm{MHz}$, keeping the ac voltage between the electrodes at $100 \mathrm{mV} \mathrm{rms}$. Ultraviolet light from a He-Cd laser $(3.82 \mathrm{eV}$, max $35 \mathrm{~mW})$ excited carriers in between the electrodes, as shown schematically in the inset of Fig. 1. The sample was cooled in a liquid-He cryostat with optical windows for the light irradiation. The temperature of the sample is controlled from $4 \mathrm{~K}$ to $270 \mathrm{~K}$ with a stability of $\pm 0.1 \mathrm{~K}$.
Figure 1 shows the temperature dependence of real parts of permittivity at $7 \mathrm{kHz}$ and $1 \mathrm{MHz}$. Open circles show the permittivity without UV-light irradiation. The permittivity has no dissipation in the frequency range and agrees well with the previous reports in $\mathrm{KTaO}_{3}$. However, once UV light is shined onto the sample, the dielectric responses change drastically; the permittivity at $4 \mathrm{~K}$ shows strong enhancement by two orders of magnitude with frequency dispersion. This photoinduced giant permittivity lasts for more than $1 \mathrm{~h}$ after stopping the light irradiation at $4 \mathrm{~K}$. The higher the temperature becomes, the faster the initial permittivity recovers. At the high temperatures over $300 \mathrm{~K}$, it takes only several seconds to recover the value, indicating that there is no permanent destruction of the sample. All samples prepared with different dimensions and electrodes exhibit the similar behavior, even when the light was irradiated from the opposite side of the electrodes.

Figure 2 shows a Cole-Cole plot of the permittivity in $\mathrm{KTaO}_{3}$ under light irradiation $\left(325 \mathrm{~nm}, 1.2 \mathrm{~W} / \mathrm{cm}^{2}\right)$ at $4.3 \mathrm{~K}$, where the imaginary parts are plotted as a function of the real part with a parameter of frequency. The inset shows their frequency dependence. Two prominent components are clearly recognizable: a semicircular dispersion in the highfrequency region and a straight-line component in the lowfrequency region. In general, the semicircle in the Cole-Cole

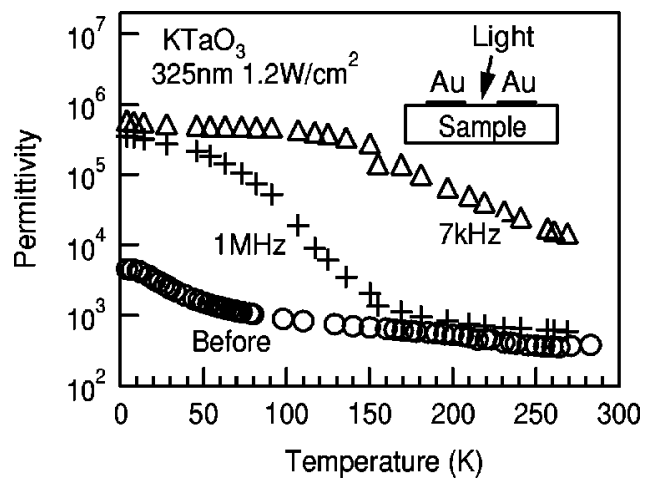

FIG. 1. Temperature dependence of the permittivity in $\mathrm{KTaO}_{3}$ before $(\bigcirc$ at $7 \mathrm{kHz}$ and $1 \mathrm{MHz}$ ) and under $(\triangle$ at $7 \mathrm{kHz}$ and + at $1 \mathrm{MHz}$ ) constant light irradiation at $325 \mathrm{~nm}$ using a He-Cd laser. The inset shows a schematic picture of the experiment and a sample with electrodes. The excitation density is $1.2 \mathrm{~W} / \mathrm{cm}^{2}$. Each measurement is performed with decreasing temperature. 


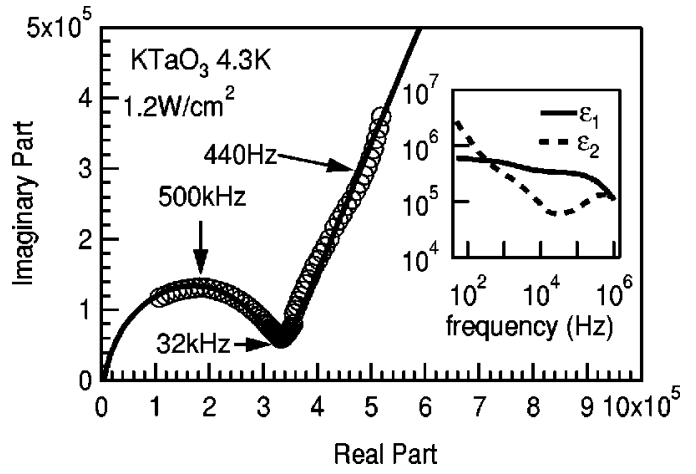

FIG. 2. A Cole-Cole plot of the permittivity in $\mathrm{KTaO}_{3}$ under laser light irradiation $(325 \mathrm{~nm})$ at $4.3 \mathrm{~K}$. The inset shows the frequency dependence of the real part (solid line) and the imaginary part (broken line). Arrows indicate the experimental data at several frequencies. A solid line is a fitting curve based on the Eq.(1) mentioned in the text, where $\epsilon_{\infty}=4800, \epsilon_{S}=3.2 \times 10^{5}, \quad \tau=2.7$ $\times 10^{-7} \mathrm{~s}, \alpha=0.13, A=1.7 \times 10^{8}$, and $\beta=0.68$.

plot indicates the existence of inhomogeneously distributed dipoles with Debye-type relaxations. ${ }^{9}$ On the other hand, the straight-line component is frequently observed in the materials with high conductance and is attributed to slowly mobile carriers, such as hopping current. ${ }^{10}$ Taking these two components into account, the following equation can reproduce the experiments:

$$
\epsilon=\epsilon_{\infty}+\epsilon_{D}+\epsilon_{C}=\epsilon_{\infty}+\frac{\epsilon_{S}-\epsilon_{\infty}}{1+(i \omega \tau)^{1-\alpha}}+A(i \omega)^{-\beta} .
$$

The first term $\epsilon_{\infty}$ is the extracted permittivity at infinite frequency. The second term $\epsilon_{D}$ phenomenologically describes the dispersions with polydispersive Debye-type relaxations. ${ }^{9}$ Here, $\tau$ is the mean dielectric relaxation time; $\epsilon_{S}-\epsilon_{\infty}$ is the oscillator strength; and $\alpha$ denotes the distribution of the relaxation time, i.e. the polydispersiveness. When $\alpha$ equals 0 , $\epsilon_{D}$ expresses monodispersive Debye-type dipoles. The third term $\epsilon_{C}$ describes the dispersion induced by the hopping current. If $\beta$ is equal to $1, \epsilon_{C}$ becomes the dc loss of free carriers. Equation (1) fits the experimental data well, as shown in Fig. 2. It should be noted that $\epsilon_{\infty}$ changes little by UV-light irradiation. Since $\epsilon_{\infty}$ is mainly governed by the soft ferroelectric mode of $\mathrm{KTaO}_{3}$, the experiment indicates that the frequency of the lowest optical phonon mode is merely changed.

We also measured the time development of the dielectric properties under constant irradiation of mild light. The photoinduced carriers have such a long lifetime that they accumulate until the density is quite significant. ${ }^{11}$ In this case, the light-irradiation time may reflect the carrier density stored in the sample if the density is not saturated. Figure 3 shows the time development of the fitting parameters in Eq. (1). A critical behavior can be found on the time development. The results are quite different before and after the time indicated by the broken line in Fig. 3. Before the threshold, the oscillator strength is proportional to the irradiation period, which eventually becomes constant after the threshold [Fig. 3(a)]. On the contrary, the relaxation rate $\tau^{-1}$ is almost

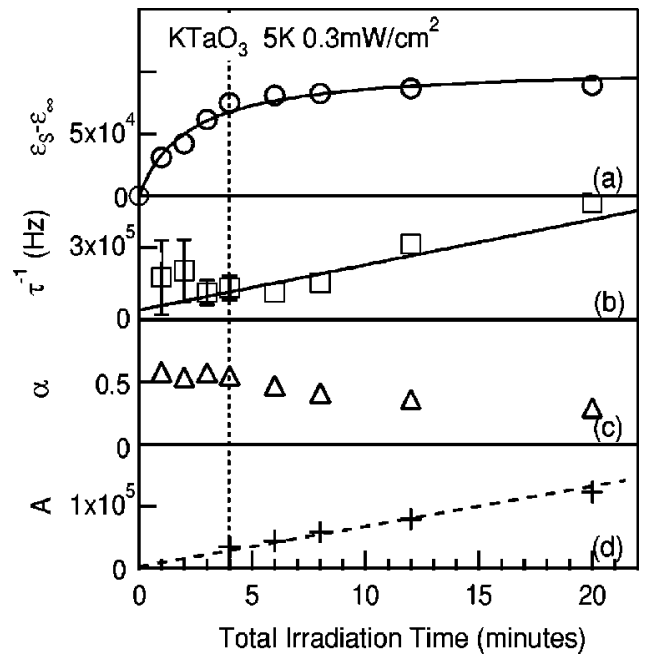

FIG. 3. Time development of (a) the oscillator strength, (b) the relaxation rate, (c) the polydispersiveness $\alpha$, and (d) the hopping current $A$. Each measurement is performed $30 \mathrm{~s}$ after stopping the light irradiation. A vertical dotted line indicates the critical time of these parameters. Solid lines are the fitting curve using the polar cluster model mentioned in the text.

constant before the threshold and then becomes proportional to the irradiation period after the threshold [Fig. 3(b)]. The hopping current term $A$ can be recognized from the threshold in the experiments, which is proportional to the irradiation period [Fig. 3(d)]. The polydispersiveness $\alpha$ decreases after the threshold [Fig. 3(c)].

The photoinduced dispersion also depends strongly on the temperature of the sample, especially the dielectric relaxation rate. The rate decreases by three or four orders of magnitude when the temperature rises from $4 \mathrm{~K}$ to $300 \mathrm{~K}$ (Fig. 4). This temperature dependence of the dielectric relaxation time is contradictory to the conventional Ahrenius-type relaxation $\left(\tau^{-1} \propto e^{-E / k T}\right)$ in the permanent dipole systems such as KLT $\left(\mathrm{K}_{1-x} \mathrm{Li}_{x} \mathrm{TaO}_{3}\right)$ crystals. ${ }^{12}$ In KLT, the relaxation rate increases when the temperature increases. We should emphasize that the difference in the temperature dependence between the photoinduced dipoles in $\mathrm{KTaO}_{3}$ and the permanent

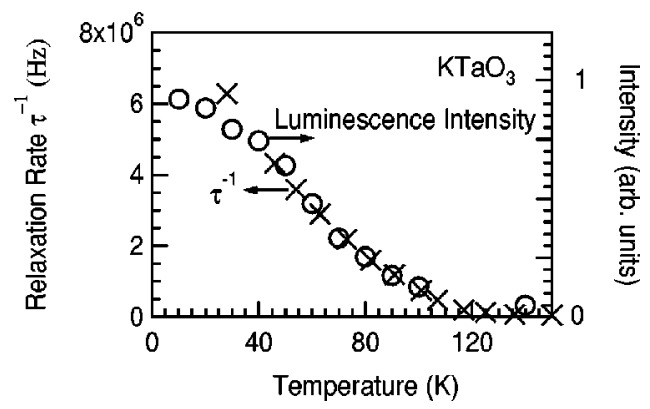

FIG. 4. Temperature dependence of the integrated luminescence intensity and the dielectric relaxation rate $\left(\tau^{-1}\right)$. The permittivity is measured under UV-light irradiation at $3.81 \mathrm{eV}$, and the luminescence spectra are measured using the third harmonics (at $4.65 \mathrm{eV}$ ) of the pulsed Ti-sapphire laser for excitation. The inset shows the typical spectra of luminescence at $4.3 \mathrm{~K}$. The luminescence intensity is integrated from $1.8 \mathrm{eV}$ to $3.5 \mathrm{eV}$. 
(a)

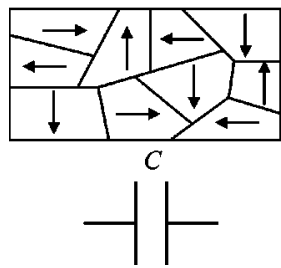

(b)

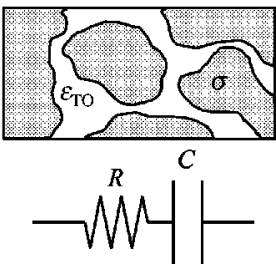

FIG. 5. Schematic pictures and equivalent circuits of (a) the polar cluster model and (b) the MW model. Arrows indicate directions of polarization in polar clusters.

dipoles in KLT shows clearly an exotic aspect of photoinduced giant permittivity. On the other hand, the oscillator strength $\epsilon_{S}-\epsilon_{\infty}$ changes a little when the temperature increases.

It is meaningful to compare the temperature dependence of the relaxation rate with that of the luminescence intensity. The luminescence intensity is proportional to the total carrier density if the system is in equilibrium. Indeed, we confirmed that the luminescence intensity depends linearly on the excitation power in our experimental conditions. In Fig. 4, the temperature dependence of the integrated luminescence intensity is plotted to compare with that of the relaxation rate. Each measurement was performed under UV light that was strong enough to saturate the oscillator strength of the photoinduced permittivity. The luminescence intensity and the dielectric relaxation rate change quite similarly as a function of the temperature. This fact strongly suggests that the carrier density is proportional to the relaxation rate of the Debyetype dispersion $\left(n \propto \tau^{-1}\right)$, which was confirmed in the timedevelopment experiment [Fig. 3(b)].

It is important to note here that the strong enhancement of the permittivity and its unconventional relaxation process should come from photoinduced carriers. We would like to propose two models to describe the physical mechanism of these exotic phenomena. One is a polar cluster model where ferroelectric clusters formed around the photoexcited carriers are responsible for the giant permittivity. The other is a Maxwell-Wagner (MW) model as discussed in the so-called high- $k$ materials. ${ }^{13}$ The MW model has also been applied to a gigantic photoinduced dielectric constant in quantum paraelectrics under a dc electric field. ${ }^{7}$ Both models consider the situation that the photoexcitation induces a kind of inhomogeneity in $\mathrm{KTaO}_{3}$.

With respect to the former model, we have already reported that the photoexcitation induce polarizationfluctuating polar clusters in $\mathrm{SrTiO}_{3} .{ }^{4}$ The photoexcited carriers form polar clusters as giving their energy to the lattice through electron-lattice interaction. In Fig. 5(a), we show the schematic picture of the model. The generated clusters can change directions of their induced permanent dipoles that obeys the following equation of motion: ${ }^{4}$

$$
\frac{d P(t)}{d t}=n b \frac{\epsilon_{T O}+2}{3} E(t)-\left(\frac{1}{\tau_{0}}-J(n)\right) P(t),
$$

where $P(t)$ represents the sum of the clusters' polarization, $J(n)$ is the interaction between polar clusters, $E(t)$ is the external electric field, $\tau_{0}$ is the bare relaxation time of the dipoles, and $b$ is the strength of the force due to the local field. Solving Eq. (2), we obtain Debye-type dispersion $\epsilon_{D}$ in Eq. (1), and the parameters become $\tau=\tau_{0} /\left[1-\tau_{0} J(n)\right], \epsilon_{S}$ $-\epsilon_{0}=n b \tau_{0}\left(\epsilon_{T O}+2\right) / 3\left[1-\tau_{0} J(n)\right], \epsilon_{0}=\epsilon_{T O}$ and $\alpha=0$.

In this model, the interaction between polar clusters $J(n)$ plays a crucial role in the exotic behavior of the oscillator strength and the relaxation time. The absence of divergence in the observed oscillator strength reveals $J(n)<0$, which suggests that the distribution for the directions of polar clusters is determined to minimize the space charges induced by polarization of these clusters (The direction of the polarization favors antiparallel between neighboring clusters). When we assume $J(n)=-J_{1} n$ under the mean-field approximation, we can successfully make a good fit of $\tau$ and $\epsilon_{S}-\epsilon_{0}$ in our experiment, shown as the solid lines in Fig. 3. The critical concentration $n_{C}=1 / \tau_{0} J_{1}$ is obtained from the fitting to be $6.3 \times 10^{17} \mathrm{~cm}^{-3}$.

On the other hand, MW model presumes that the photoexcitation induces metallic clusters and the thin nonconducting layers to separate them. The net photon number at the critical density, $n_{C}=6.3 \times 10^{17} \mathrm{~cm}^{-3}$, is near the Mott density of the insulator-metal transition in $\mathrm{KTaO}_{3} .{ }^{14}$ It is therefore reasonable to consider that the strong enhancement of the permittivity comes from the precursory behavior of the insulator-metal transition. The metallic clusters grow as the carrier density increases and their wave functions overlap each other. According to the Hall mobility experiment, ${ }^{15}$ the major carriers of the photocurrent in $\mathrm{KTaO}_{3}$ are electrons. This means that holes should be trapped deeply and they merely recombine with electrons. This induces stable-free electrons and lead to the long lifetime of the metallic clusters. A plausible candidate for the deep hole centers is a transition-metal impurity whose valence changes via light irradiation, like-rare earth ions in photostimulable phosphors.

In MW model, we assume that the metallic clusters have conductivity $\sigma$. Around the clusters there are nonconducting layers with dielectric constant $\epsilon_{T O}$ similar to the dielectric constant in $\mathrm{KTaO}_{3}$ without irradiation. Figure 5(b) describes the situation, which is equivalent to the 3D-MW model. ${ }^{13}$ For simplicity, we discuss an equivalent circuit of the 1D-MW model as shown in the equivalent circuit of Fig. 5(b). The metallic clusters are collectively transposed to the resistance $R$, and the volume ratio of the nonconducting layers determines the capacitance $C$ in the circuit. In this case the observed permittivity shows the Debye-type dispersion $\epsilon_{D}$ in Eq. (1) as $\epsilon_{S}-\epsilon_{0}=\epsilon_{T O} / \gamma$ and $\tau=\epsilon_{T O} / \gamma \sigma$. Here, $\gamma$ is the constant ratio of the effective thickness of the nonconducting layers to that of the conducting layers. The inverse of $\gamma$ describes the enhancement factor by light irradiation. For example, $\gamma$ should be 0.01 in the case shown in Fig. 2. One can immediately obtain the relation that $\tau$ is inversely proportional to the carrier density (because $\sigma \propto n$ ) and that the oscillator strength is a constant.

Assuming that the Mott density for the insulator-metal transition should fluctuate spatially, we can describe the critical behavior with the MW model. In $\mathrm{KTaO}_{3}$, spatial inhomogeneity of the permittivity should be induced by inevitably inherent impurities or defects in QPE. The Mott critical den- 
sity, therefore, would be spatially inhomogeneous, because it is inversely proportional to the cubed permittivity. Local aggregation of the impurities or defects should bring about a region whose permittivity is smaller than the rest of the sample. The region may have an anomalously high Mott density and be left nonconducting even when the carrier density becomes high. The effective thickness of the nonconducting layers changes little in this density region. Otherwise, when the carrier density is low enough, metallic clusters grow and the volume ratio of the nonconducting region gradually decreases as the carrier density increases.

Whatever be the microscopic picture of the clusters, it is revealed that the light irradiation induces mesoscopic clusters in $\mathrm{KTaO}_{3}$. In order to distinguish whether the clusters are metallic or polar, we need further information on the dielectric constants at higher frequencies, typically $\mathrm{THz}$ region where the soft-ferroelectric mode or the plasma frequency may exist. We are performing this experiment at present. At the moment, we cannot conclude which model is more reasonable for the photoinduced giant permittivity in $\mathrm{KTaO}_{3}$. In fact we cannot distinguish local current described in the MW model from displacement current in the classical
Maxwell equation. Furthermore, it is worth noting that the understanding of the nature of these clusters may shed a new insight onto the mechanism of the high- $k$ materials that also have the giant permittivity.

In conclusion, the photoinduced giant permittivity in $\mathrm{KTaO}_{3}$ was observed and attributed to the metallic or polar clusters formed by the excited carriers. The critical density of the enhancement has been confirmed from the experiments. The exotic behaviors observed in the relaxation process can be consistently interpreted by considering the change of the carrier density. The large fluctuation originated from the quantum paraelectricity is presumable to induce the mesoscopic inhomogeneity and the giant permittivity. It is elucidated that the novel macroscopic phase in the excited state is induced by band-to-band excitation in $\mathrm{KTaO}_{3}$, but further experiments are needed to confirm our model.

The authors thank Mr. T. Hasegawa for fruitful discussion, and Dr. M. Nagai for critical reading. This work was partially supported by the Asahi Glass Foundation, and a Grant-in-Aid for Scientific Research (B) from Japan Society for the Promotion of Science (JSPS).
*Electronic address: ikufumi@scphys.kyoto-u.ac.jp

†UL: http://www.hikari.scphys.kyoto-u.ac.jp/e_home.html

${ }^{1}$ Relaxations of Excited States and Photo-Induced Structural Phase Transitions, edited by K. Nasu (Springer-Verlag, Berlin, 1997).

${ }^{2}$ T. Tayagaki and K. Tanaka, Phys. Rev. Lett. 86, 2886 (2001).

${ }^{3}$ V.S. Vikhnin, R.I. Eglitis, S.E. Kapphan, E.A. Kotomin, and G. Borstel, Europhys. Lett. 56, 702 (2001).

${ }^{4}$ T. Hasegawa, Y. Yamada, S. Mouri, and K. Tanaka, J. Phys. Soc. Jpn. 72, 41 (2003).

${ }^{5}$ S.A. Prosandeev, V.S. Vikhnin, and S.E. Kapphan, J. Phys.: Condens. Matter 14, 4407 (2002).

${ }^{6}$ G.P. Banfi, P. Calvi, P. Camagni, E. Giulotto, L. Rollandi, G. Samoggia, and P. Sangalli, J. Phys.: Condens. Matter 9, 507 (1997).

${ }^{7}$ M. Takesada, T. Yagi, M. Itoh, and S. Koshihara, J. Phys. Soc. Jpn. 72, 37 (2003).

${ }^{8}$ D.M. Hannon, Phys. Rev. 164, 366 (1967).
${ }^{9}$ K.S. Cole and R.H. Cole, J. Chem. Phys. 9, 341 (1941).

${ }^{10}$ A.A. Bokov, M.M. Kumar, Z. Xu, and Z.G. Ye, Phys. Rev. B 64, 224101 (2001).

${ }^{11}$ I. Katayama, Y. Ichikawa, and K. Tanaka (unpublished). The luminescence intensity in $\mathrm{KTaO}_{3}$ decays as $\propto t^{-1}$ in the range from $100 \mathrm{~ns}$ to $1 \mathrm{~s}$ at $4 \mathrm{~K}$, indicating that the photoexcited carriers are localized and are accumulated in the sample. The intensity decreases when the temperature increases, while the decay profile unchanges.

${ }^{12}$ P. Doussineau, Y. Farssi, C. Frénois, A. Levelut, K. McEnaney, J. Toulouse, and S. Ziolkiewicz, Phys. Rev. Lett. 70, 96 (1993).

${ }^{13}$ I.P. Raevski, S.A. Prosandeev, A.S. Bogatin, and M.A. Malitskaya, cond-mat/0209015 (unpublished).

${ }^{14}$ P.A. Cox, Transition Metal Oxides (Oxford University Press, New York, 1992), p. 195.

${ }^{15}$ K. Ohi, Y. Takeda, and Y. Ohata, Izv. Akad. Nauk SSSR Ser. Fiz. [Bull. Acad. Sci. USSR, Phys. Ser. 41, 129 (1977)]. 